\title{
AN INTRODUCTION OF BLACK-BOX IN AUTOMOBILES
}

\section{B. S. MAHANTO* \& DEBASISH SARAN}

Institute of Technical Education and Research, Siksha 'O'Anusandhan Deemed to be University, Odisha, India

The essential inspiration driving this paper is to develop a model of the Vehicle Black Box System VBBS that can be brought into any vehicle wherever all through the world. This model can be sketched out with the least number of circuits. The VBBS can add to growing progressively verify vehicles, improving the treatment of mishap losses, assisting protection organizations with their vehicle crash examinations, and upgrading street status so as to diminish the demise rate. Due to its good conditions and demand of time. It empowers examination to gather just as it will be some help to the protection offices. Also, it will help to those exemplary drivers who are deceptively blamed for setback cases. We can use this structure for emergency disaster alert too. At the point when the car accidents the system send the setback alert in nearest salvage administration by giving the present position of the vehicle to a pre-customized versatile number by methods for GSM modem.
\end{abstract}

KEYWORDS: Microcontroller (PIC18F452), GPS, GSM, LCD Display \& Regulated Power Supply

Received: Apr 02, 2019; Accepted: Apr 23, 2019; Published: Jun 13, 2019; Paper Id.: IJMPERDJUN2019186

\section{INTRODUCTION}

In this paper, we have a tendency to project the GPS (Global Positioning System) /GSM (Global System for Mobile Communication) for driver facilitate and automobile perception. Measuring device and GPS following structure is formed for screen the disaster[1]. The system includes pleasant components GPS device and GSM module. Within the event of an incident, if any hurt happened to the automobile driver or explorers thus perhaps there'll be loss of lives in light-weight of delay in therapeutic facilitate [2]. Keeping this idea in our mind, we have a tendency to area unit proposing a structure wherever automobile itself insight the strain emergency advantage for brisk reaction if there got to emerge an occurrence of mishap or any emergency scenario. once the incident, this remote gizmo can send portable short message showing the circumstance of auto by GPS system to relatives, nearest police home base and specialist's offices[3]. The emergency therapeutic organization (EMS) is given to the driving force. The farthest purpose estimation is employed to decide on speed of bicycle and fall or natural event dynamically. The endeavour works pleasingly logically, will discover the vehicle travel territories as line of longitude, scope with the area for offer and take no more than half-dozen meters from the veritable zone. This technique what is more logs the data like speed, most outstanding speed and partition data of the vehicle [4].

\section{Objectives}

- $\quad$ Read, records and shows the scope and longitudes of the Automobile.

- $\quad$ Speed of the Automobile.

- Add up to remove gone by the Automobile. 
- Most extreme speed of the Automobile all through the voyage.

- Number of ends taken by the Automobile all through the excursion.

\section{METHODOLOGY}

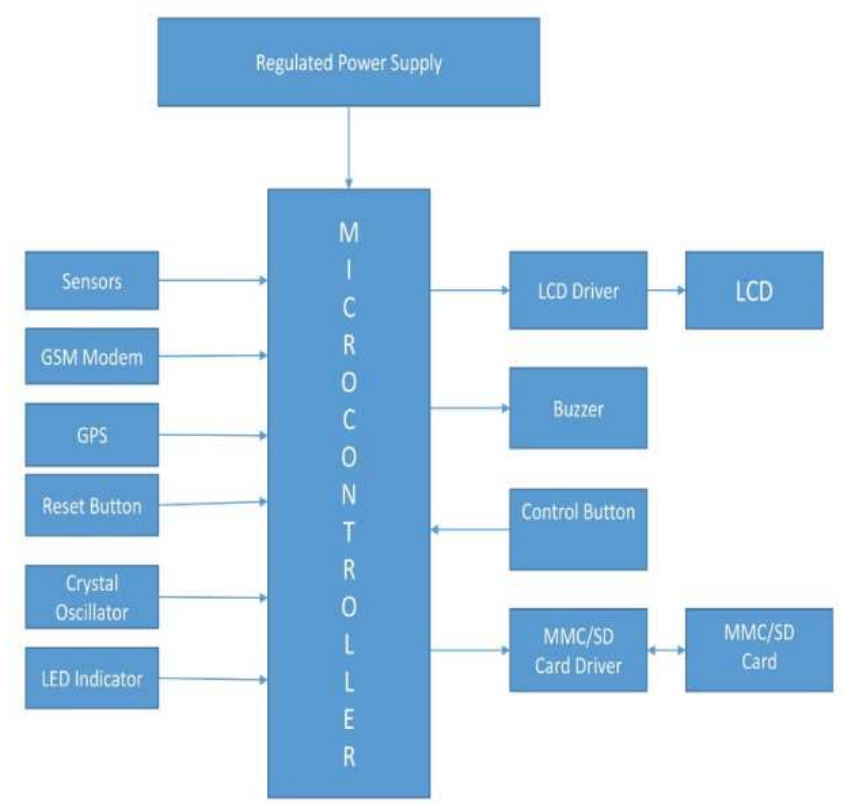

Figure 1: Block Diagram of Black Box System in Vehicle

- Power distribution can be a source of power, which arrangements identifying or electrical types of essentialness or building of weights is popularly called PSU or an electricity source device.

- The Global Positioning System (GPS) can be really just a booming advancement, that supplies versatility of organizing to track, appearing right into and GIS info set [5].

- Even the GPS supplies organizing that is three dimensional that is constant 24 hrs. Every day throughout the whole world. After the client's standing was settled, the GPS device may figure information, for example, rate, bearing, track, vacation escape.

- A winner of one of the contraptions would be LCD show up. Debut is utilized within this errand. This consists of 16 personalities for every single line by two lines [6].

- GSM will ship the SMS as soon as a collision occurs. GSM can be an electronic platform that a pace of $270 \mathrm{kbps}$. For GPS lasted into the micro controller and also are certain to find the advice. Information will be perused by Control and reveal it. In case any injury transpires GSM will ship the SMS into the dog master of this vehicle [7].

- A memory is a solid state digital memory information Demo contraption fit.

- Drive nets have been utilized to restrain some advantage a little piece of another strategy or this system. Inch.

Power-supply: At the ability to provide you, LPC2148 performs on about a few.3 Cable electricity offers. Lumen can be used for creating three-. $3 \mathrm{~V}$ distribution. Fundamental peripherals Motor Driver IC, like display will work on 5V. Thus AC mains provide you is now regenerated right into 5V. Lumen Seventy-Five is used to Transform 5V to a Few.3V. 
Electrical Electricity deal was made by [8]

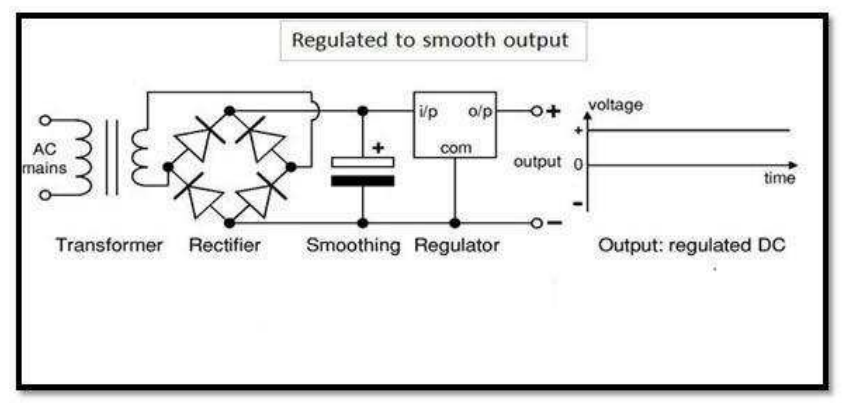

Figure 2

- Transformer: it is familiar with measure $230 \mathrm{~V}$ AC to $9 \mathrm{~V}$ AC provide you down. It gives isolation among gate AND electricity grids. Rectifier: it truly is utilized to change a-c supplies. [9]

- Reset Circuit: Re-set BUTTON: This is crucial inside the device to draw the system back into the manner and to prevent programming disadvantages.

- OSCILLATOR: Oscillations are supplied with a crystal plus are all essential for that platform.

- $\quad$ At ARM7 loved ones, LPC2148 is broad utilized IC. ARM chip relies on low instruction set computing (RISC) designs. ARM'S schooling collection is adhered and also uniform. Ten two-bit ARM processors include an instruction put: overall 32-bit ARM instruction group, and 16-bit Thumb instruction collection. It supports pointpipe liner within a straight-forward 3-stage pipeline, so the instructions follow through up 3 levels: decipher, Seize and Execute. [10]

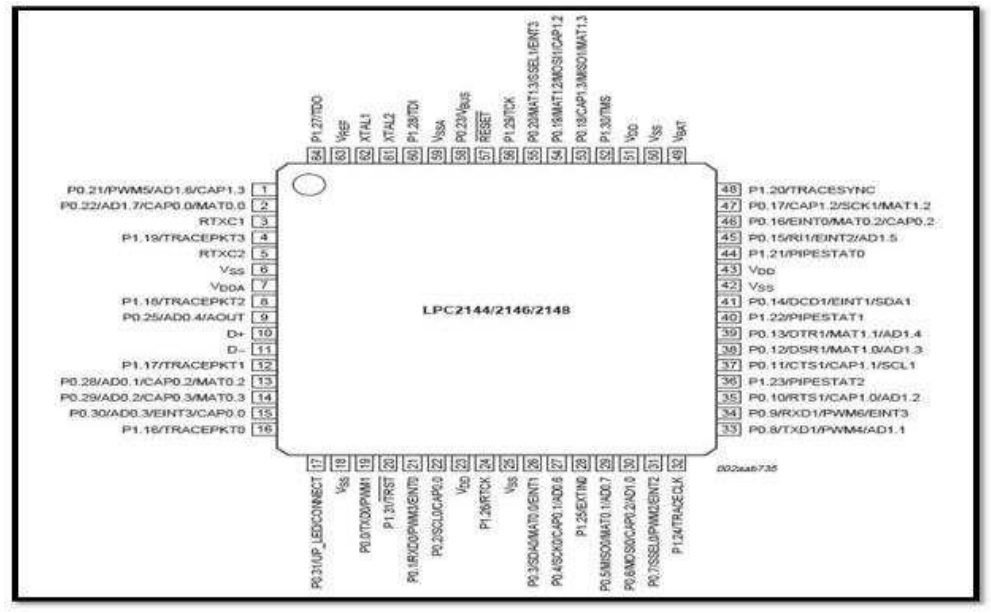

Figure 3

\section{Temperature Sensor (LM75)}

Even the temperature comprises a Delta Sigma analog- to-digital apparatus, and also also a over-temperature sensor. That the LM75 will be questioned by the server by way of its port to automatically navigate equilibrium. Opendrain in excess of heating output signal (OS) sinks present when the cushioned temperature limitation is surpassed. Even the OS out-put works in both manners, compactor or interrupt [11]. 


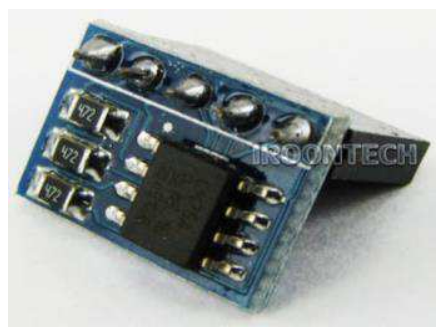

Figure 4

Powerup is currently in compact style using the defaults of Tryst along with TOS. Even the $3.0 \mathrm{~V}$ to 5.5 squat present you voltage fluctuates, very low deal recently, and I2C interface assemble up the LM75 well suited for many software in thermal direction and security.

Filter: it is won't to cut dilemma of DC output from the rectifier complete. Regulator: it truly is won't to govern DC features output

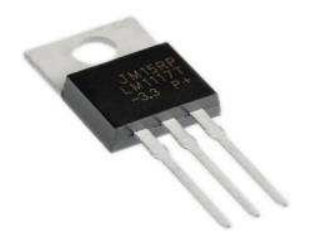

Figure 5

\section{BELT SENSOR}

The belt is sensing part signals whether chair straps step that is square mended. We've obtained applied Audio Jack is a Belt detection ingredient. That is guaranteed by assessing whether the switch is ironed or not. One particular push can be utilized to watch the place of their entire belt across the driveway. The drive is set on existence belt also will be offering sense 'zero' after the buckle is logic and employed 'you' when the buckle is not set from the force [11]

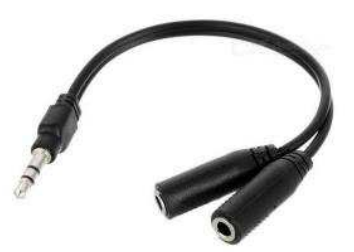

Figure 6

\section{Door Sensor (Leaf Switch)}

Inside our case we are apt to make use of foliage switch for a do or detection component to be sure whether the doorway is shut or shut. While this foliage swap is attached, it features the logic 'a single' shows which way the door is shut of course, whether or not it features the logic 'zero' this means foliage switch has been split out of another and doorway remains available. 


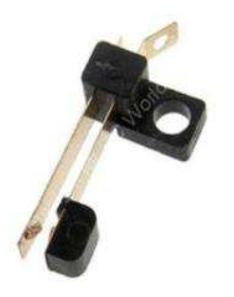

Figure 7

\section{RTC (Real Time Clock)}

Authentic clock (RTC) since the identify urges our clock module. The DS1307 clock IC is currently Associate at Nursing an eight-pin system manipulation Associate. The clock /calendar provides seconds, hours, hours, date, day, month and month comprehension that are capable.

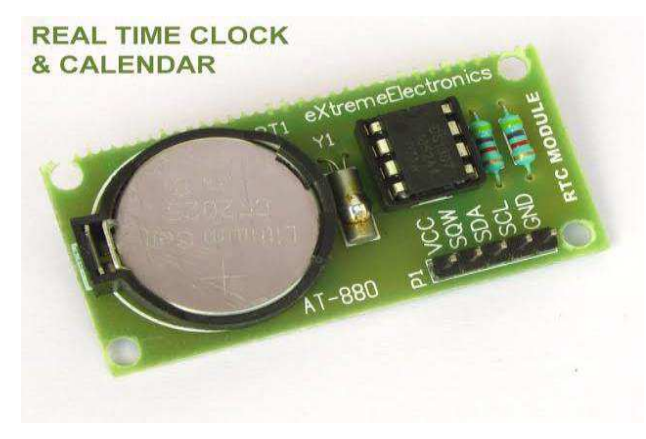

Figure 8

\section{OPTOCOUPLER}

Inside our case, we have a tendency to utilized MOC7811 as affiliate on coupling at Nursing to identifying wheels' rate. This on coupling module together with Associate at a photo diode and Nursing IR cage installed onto this. That is utilized being a spot setting component change to hunt the set of this wheel from. Photo diode mounted deal with another and also it is made of both IR crystal rectifier and shut human anatomy. Slice on the circuit out of diode that is icon and crystal rectifier, we have a tendency to utilize disrupt to figure out this wheel's rotation rate.

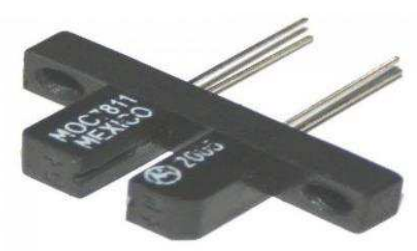

Figure 9

\section{RS232}

Rs-232 is the fact the conventional sequential communicating system that's required to transport info |the date |the info\} from micro control on public pc (personal computer) by way of some kind of details wires. Rs-232 and Its trap arrangement's interfacing into the control manipulation MAX232. 


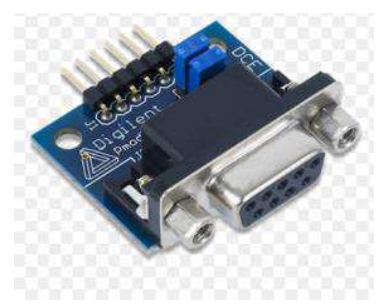

Figure 10

\section{DATA RETRIEVING FROM EEPROM}

EEPROM might be some type of computing device's processor which keeps whilst maybe perhaps not needing an effect offer, maintain advice.

Every depth of this part is figured Whilst the injury come about and also stay in memory. All these principles really are valuable to comprehend the incident. This deep understanding of this mishap will be great to this insurance policy carrier and for authorities. Even as we often to find the worth or information in products manipulation cable connects to a personal computer that was non-public that. All these I2C-compatible electrically efface in a position programmable memory (EEPROM) apparatus square step coordinated as eight192 x 8 pieces (M24C64) along with 4096 $\times$ 8 pieces (M24C32). I2C employs a port, containing also a clock lineup plus a knowledge line. The apparatus to execute a key 4-bit Apparatus type emblem code (1010) in compliance using all the I2C bus expression.

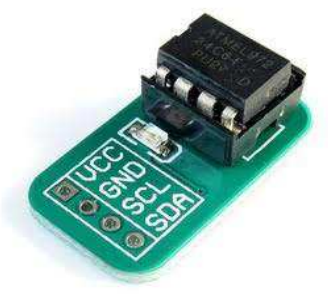

Figure 11

\section{PROPOSED APPROACH}

In this part, our system is we have to generate two upgrades. These upgrades step of security choices inside the gear process that is documenting such as - -infra-red detection part.

- $\quad$ gasoline detector (smoke sensor)

- Infra-red sensing element:

The detection part is really to endure exactly at the space. Sensing part generate high-frequency undulation affiliate at Nursing gauge that the heat being secreted detective job motion and by means of an item that's obtained endorsed from the part. Its space sensing fluctuate is two $\mathrm{cms}$ into $3 \mathrm{~cm}$ [12].

\section{Gasoline Sensing Component (Smoke Detector)}

A petrol sensor might be described as considering a system which finds the existence of electrons within a neighborhood, commonly like an element of the stability apparatus. Therefore |this sort $\}$ of devices can be utilized to detect a petrol flow and also port using a consequence system thus a system could be automatically near up [13,14]. 
- $\quad$ Sensitive to LPG, ISO butane, Propane.

- Sensitive to alcohol, smoke.

- Quick response.

- Stable and long life.

- $\quad$ Easy drive circuit.

\section{CONCLUSIONS}

As there are various focal points of presenting black box in cars. Vehicle black box are not exactly equivalent to the thoughtful that is in planes, which always record sound, mechanical limits and zone. In automobiles, the crate just transmits information to the auto's PC in the event of a mishap or air pack association. Recorders get vehicle speed, brake activity, crash controls and even seat strap use at the period of the event. In future the Automobile Black Box will be the fused bit of a vehicle. In light of its good conditions and demand of time. It empowers examination to bunch just as it will be some help to the protection offices. Moreover, it will help to those innocent drivers who are deceptively blamed for setback cases.

\section{Future Scope}

- This project will have utilized in various kind of vehicles and furthermore utilized for security reason.

- Use for vehicle safety.

- Accident investigation.

- Use in Insurance company.

\section{REFERENCES}

1. V. Pagadala, S. Rani, and B. K. Priya, "Design and Implementation of the Prevention and Analysis of the Accident for Automobiles," in 2018 International Conference On Advances In Computing, Communications And Informatics (ICACCI), 2018, pp. 2283-2289.

2. P. Kulkarni, P. K. Rajani, and K. Varma, "Development of On Board Diagnostics (OBD) Testing Tool to Scan Emission Control System," in 2016 International Conference On Computing Communication Control And Automation (Iccubea), 2016.

3. W.-H. Kim, K. Yi, and C.-M. Kyung, "Energy-efficient illumination-invariant change detection in DCT coefficient domain for vehicular black box camera," Electron. Lett., vol. 51, no. 11, May 2015.

4. C. Mohan, H. G. Elizabeth, P. C. Navya, and V. Bagyaveereswaran, "Electronic Scrutinizer for Automobiles," in 2015 International Conferenced On Circuits, Power And Computing Technologies (ICCPCT-2015), 2015.

5. W. M. Kang, J. D. Lee, and J. H. Park, "VBSM: VCC-Based Black Box Service Model with Enhanced Data Integrity," Information, vol. 5, no. 4, pp. 526-534, Dec. 2014.

6. M. J. Prasad, S. Arundathi, N. Anil, Harshikha, and B. S. Kariyappa, "Automobile Black Box System for Accident Analysis," in 2014 International Conference On Advances In Electronics, Computers And Communications (ICAECC), 2014. 
7. Bosco, S. J., Wolfgang, N., Bonaventure, D., \& Tibi, B. (2015). Disassemblability and Reassemblability Parameters Analysis: Automobile Maintenance Context Study.

8. K. Kim, K.-Y. Choi, and J.-W. Lee, "Fault Localization Method by Partitioning Memory Using Memory Map and the Stack for Automotive ECU Software Testing," Appl. Sci., vol. 6, no. 9, Sep. 2016.

9. Ehiabhili, J., Ezeh, C., \& Orji, O. (2018). Single Phase Microcontroller-Based Automatic Changeover Switch. International Journal of Electronics, Communication \& Instrumentation Engineering Research and Development (IJECIERD), 8(1), 7-16.

10. H.-M. Moon, K.-H. Kim, M. Lee, P. Kim, and S. B. Pan, "A Study on the Context Aware System for Intelligent Automobile Black Box,” in 201527 TH Chinese Control And Decision Conference (CCDC), 2015, pp. 2536-2540.

11. S.-K. Lee, J.-W. Lee, D.-S. Yun, and O.-C. Kwon, "Development of Reusable In-Vehicle Component For Vehicle-IT Application," in 2011 6TH International Conference On Computer Sciences And Convergence Information Technology (ICCIT), 2012, pp. 77-80.

12. M. Contag et al., "How They Did It: An Analysis of Emission Defeat Devices in Modern Automobiles," in 2017 IEEE Symposium On Security And Privacy (SP), 2017, pp. 231-250.

13. C. Kim, D. Shin, D. Shin, and C.-N. Yang, "Secure protection of video recorder video in smart car," Int. J. Distrib. Sens. NETWORKS, vol. 12, no. 12, Dec. 2016.

14. C. Patil, Y. Marathe, K. Amoghimath, and S. S. David, "Low Cost Black Box for Cars," in 2013 Texas Instruments India Educators' Conference (TIIEC 2013), 2013, pp. 49-55.

15. S. Zaglauer and M. Deflorian, "Multi-criteria Optimization for Parameter Estimation of Physical Models in Combustion Engine Calibration," in Evolutionary Multi-Criterion Optimization, EMO 2013, 2013, vol. 7811, pp. 628-640.

16. W. A. Young II and G. R. Weckman, "Using a heuristic approach to derive a grey-box model through an artificial neural network knowledge extraction technique," NEURAL Comput. Appl., vol. 19, no. 3, pp. 353-366, Apr. 2010. 